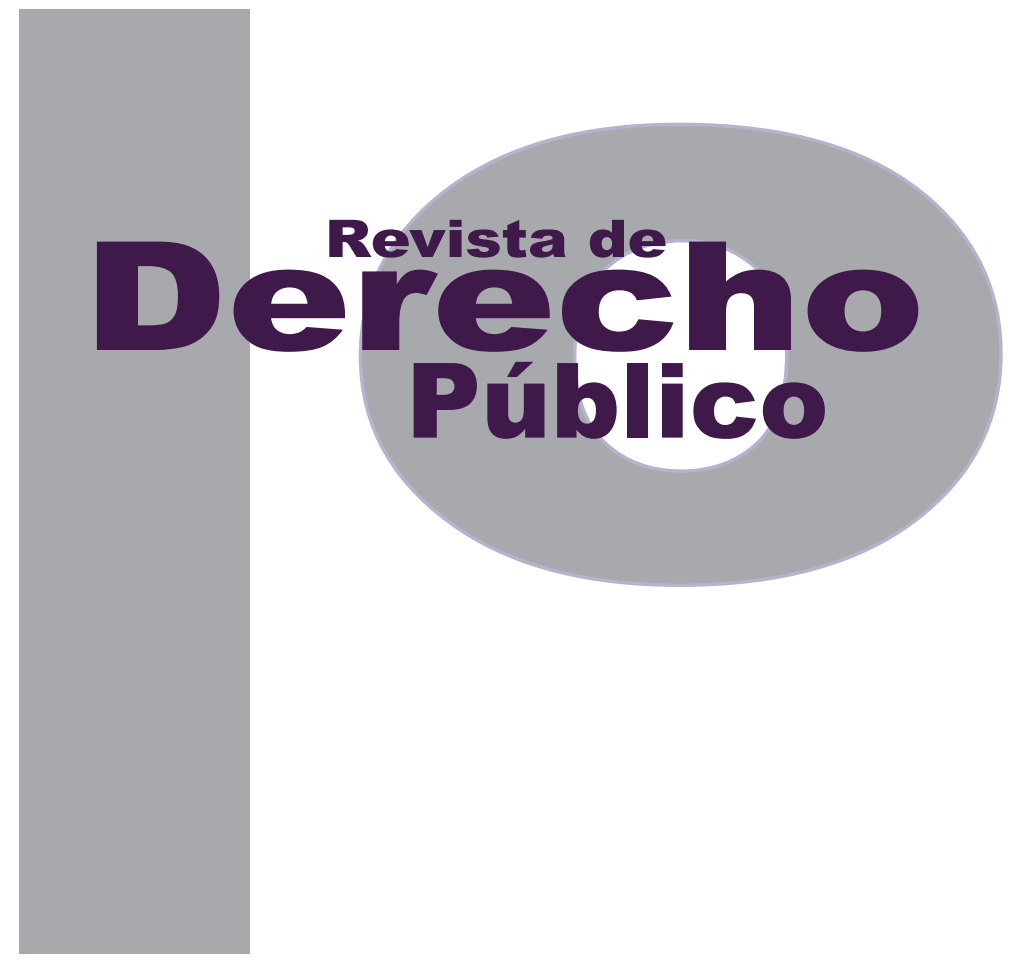

\title{
MOdeloS De GeSTIÓN: HACIA UN NUEVO DERECHO PROCESAL ORGANIZACIONAL
}

\author{
LINA SANTOS
}

Revisión de tema

Universidad de los Andes

Facultad de Derecho

Revista de Derecho Público N. ${ }^{\circ} 32$

Enero - Junio de 2014. ISSN 1909-7778 


\section{Modelos de gestión: hacia un nuevo derecho procesal organizacional Resumen}

Este trabajo se concentra en hacer, por un lado, una exposición detallada de la discusión sobre los modelos de gestión de los despachos judiciales y, por otro lado, proponer en Colombia el surgimiento de un nuevo derecho procesal organizacional que se encargue de ellos. De esta manera, se pretende generar una base de conocimiento que contribuya a la difusión de este asunto y que demuestre la importancia de crear una rama del derecho que se ocupe de su estudio y aplicación. El anterior cometido se realiza a través de la revisión de la literatura relacionada con modelos de gestión de los despachos judiciales y de gerencia judicial que permitirá desarrollar un ejercicio descriptivo de los contenidos más relevantes sin dejar de lado un enfoque crítico a la hora de interpretarlos. Así las cosas, se evidencia la verdadera finalidad de la gerencia judicial, la importancia de incluir a los funcionarios judiciales en el debate y la necesidad de agilizar la discusión sobre este aspecto en Colombia, entre otros.

Palabras clave: Modelos de gestión de los despachos judiciales, gerencia judicial, administradores judiciales, derecho procesal organizacional, servicios comunes, eficiencia judicial.

\section{Management models: Towards a new organizational procedural law}

\section{Abstract}

This paper focuses in a detailed description of the discussion on the management models of judicial offices and suggests the emergence of a new organizational procedural law that takes care of them. Thus, it aims to create a knowledge base that contributes in the difussion of this issue and demonstrates the importance of creating a new branch of law that deals with its study and application. The above objective will be carried out through a review of the literature related to management models of judicial offices and judicial management in order to develop a descriptive exercise of the relevant contents without leaving behind a critical approach when interpreting them. In this context, the real purpose of judicial management, the importance of including judicial officers in the debate and the need to expedite the discussion in Colombia, among others, will emerge.

Keywords: Management models of judicial offices, individual court management, judicial administrators, organizational procedural law, judicial common services, judicial efficiency.

\section{Modelos de gestão: para um novo direito processual organizacional Resumo}

Este trabalho concentra-se em fazer, por um lado, uma exposição detalhada da discussão sobre os modelos de gestão dos despachos judiciais e, por outro lado, propor na Colômbia o surgimento de um novo direito processual organizacional que se encarregue deles. Desta maneira, pretende-se gerar uma base de conhecimento que contribua para a difusão deste assunto e que demonstre a importância de criar um ramo do direito que se ocupe de seu estudo e aplicação. $O$ anterior cometido realiza-se através da revisão da literatura relacionada com modelos de gestão dos despachos judiciais e de gerência judicial que permitirá desenvolver um exercício descritivo dos conteúdos mais relevantes sem deixar de lado um enfoque crítico na hora de interpretá-los. Assim, evidencia-se a verdadeira finalidade da gerência judicial, a importância de incluir os funcionários judiciais no debate e a necessidade de agilizar a discussão sobre este aspecto na Colômbia, entre outros.

Palavras-chave: Modelos de gestão dos despachos judiciais, gerência judicial, administradores judiciais, direito processual organizacional, serviços comuns, eficiência judicial. 


\section{SUMARIO}

Introducción - I. DISCUSIÓN CONCEPTUAL DE LOS MODELOS DE GESTIÓN - II. CONVERGENCIAS HACIA UN NUEVO MODELO DE GESTIÓN - III. COLOMBIA EN EL DEBATE - IV. CONCLUSIONES - Bibliografía comentada. 


\title{
Modelos de gestión: hacia un nuevo derecho procesal organizacional ${ }^{1}$
}

\author{
Lina Santos ${ }^{2}$
}

Introducción

Actualmente, la administración de justicia colombiana está pasando por una de sus peores crisis: padece de falta de confianza por parte de los ciudadanos, de altos niveles de congestión e impunidad, de mala calidad en la prestación del servicio, de pésima infraestructura física, de ausencia de una política pública coherente, de baja seguridad jurídica debido a la variabilidad de los autos y sentencias y de falta de una entidad que realmente gestione esta rama del poder público y tenga voluntad política para solucionar sus problemas. ${ }^{3}$ No en vano, la última encuesta presentada por Transparencia Internacional, en julio de este año, revela que la justicia es una de las instituciones más corruptas del país, ya sea porque se soborna al operador jurídico para

Cómo citar este artículo: Santos, L. (Junio, 2014). Modelos de gestión: hacia un nuevo derecho procesal organizacional. Revista de Derecho Público, 32.

2

Estudiante de Derecho de la Universidad de los Andes, próxima a graduarse. Correo: Lm.santos443@uniandes.edu.co

3 acelerar el proceso y los trámites o porque "era la única manera de obtener el servicio" (El Tiempo, 2013).

A pesar de esta evidente crisis, las últimas reformas judiciales relevantes (particularmente las leyes 794 de 2003 y 1395 de 2010) no han alcanzado los resultados esperados y han demostrado no contemplar los mecanismos suficientes para resolver los principales problemas de la rama. Como consecuencia de ello, los índices de congestión, impunidad, calidad y percepción ciudadana no han mejorado y, por el contrario, parecen empeorar cada día más. ${ }^{4}$

En este sentido, si las distintas reformas implementadas no han alcanzado los resultados esperados y no han contrarrestado las debilidades del sistema, ¿en qué fallan los encargados del diseño de las políticas públicas dirigidas a atender la crisis administrativa que evidencian los despachos judiciales?, ¿por qué el sistema 
judicial colombiano cada día aparenta estar en peores condiciones para atender la demanda de justicia existente? Estas son algunas de las preguntas que surgen al constatar la realidad del sistema de administración de justicia. Una de las primeras respuestas es la falta de modelos de gestión de los despachos judiciales, razón por la cual es indispensable cambiar el enfoque de las reformas y estudiar a nivel micro dónde están las principales causas de ineficiencia y congestión de la rama judicial.

Si se analizan las últimas reformas al sistema (Ley 794 de 2003, Ley 1395 de 2010 y el Código General del Proceso, entre otras), se observa que estas se han concentrado principalmente en aspectos procesales o en asuntos macro: creación o supresión de procesos, acortamiento de términos procesales, creación de nuevos jueces temporales o de descongestión, implementación de la oralidad, etc. Pocas veces, por no decir ninguna, se ha hecho mención del tema de la microgestión o microgerencia del despacho judicial, es decir, todo lo relativo a la organización del personal dentro del juzgado, a la definición y ejecución de las funciones y los recursos, al manejo de casos, a la atención de baranda, a la implementación de tecnologías de la información y la comunicación (TIC), entre otros.

Si se tiene en cuenta que el derecho procesal es el conjunto de normas "que tienen por objeto y fin la realización del derecho objetivo, a través de la tutela del derecho subjetivo, mediante el ejercicio de la función jurisdiccional" (Morales, 1965, p. 10), es evidente que se quedó corto frente a la inclusión de los modelos de gestión como herramienta para alcanzar el derecho objetivo, porque no es posible pensar en la obtención de justicia material si las normas procedimentales no se complementan con reglas que garanticen la eficiencia de la administración de justicia. Por esta razón, el derecho procesal, entendido como conjunto de normas jurídicas que regulan el ejercicio de la función jurisdiccional del Estado (Morales, 1965, p. 9), también debe incluir conceptos de gerencia judicial. Por ello, quizás sea hora de pensar en la creación de un nuevo derecho procesal organizacional ${ }^{5}$ cuyo estudio esté concentrado en la gerencia y modelos de gestión de los despachos judiciales como mecanismos para garantizar el derecho subjetivo.

En este orden de ideas, el presente artículo de revisión de tema se concentra, primero, en hacer una exposición detallada de la discusión sobre los modelos de gestión de los despachos judiciales; segundo, en proponer el surgimiento de un nuevo derecho procesal organizacional que se encargue de ellos, pues, finalmente, el despacho judicial, al igual que cualquier organización, requiere de una gestión correcta y técnica para alcanzar los objetivos que se propone.

Es por esto que el objetivo principal de este documento es responder la pregunta ¿qué se ha

5 El término derecho procesal organizacional surgió en Argentina y no ha sido muy difundido en otros países. Ver: Garavano, G. C., Chayer, H. M., Cambellotti, C. A. y Ricci, M. (1999) y Quiroga Lavié, H. (1998). Personalmente, no me termina de convencer el concepto, ya que considero que puede acabar subyugado al derecho procesal formal. No obstante, inicialmente puede ser una buena manera de llamar la atención de los procesalistas, quienes normalmente le dan la espalda a estos asuntos. 
escrito sobre modelos de gestión de los despachos judiciales en el ámbito internacional y local y cómo se enmarca Colombia dentro de esa discusión? Para ello se expone la información más relevante y actual sobre la discusión en torno a los modelos de gestión de los despachos judiciales, como materia de estudio de un nuevo derecho procesal organizacional, para contribuir en la difusión de su conocimiento y para permitir que cualquier persona interesada en informarse sobre este asunto pueda hacerlo de una manera sencilla y completa, sin tener que padecer las dificultades que genera la dispersión de la información.

El anterior cometido es un ejercicio más complicado de lo que parece, si se tiene en cuenta que los debates alrededor de este tema son novedosos y, en consecuencia, ha habido poca producción literaria y la que existe no ha tenido mucha difusión. Por esta razón, la compilación de la información y la creación de una base de conocimiento es un aporte valioso al desarrollo de este tema que, además de permitir conocer el estado actual de la discusión y el rumbo al que se dirige, posibilita extraer unas conclusiones sobre la manera como se ha abordado este asunto y sobre algunas de sus debilidades.

Para llevar a cabo este propósito, se empieza por hacer una revisión de la literatura relacionada con modelos de gestión de los despachos judiciales y gerencia judicial. Esta parte incorpora algunos de los resultados de la investigación realizada por Juan Enrique Vargas Viancos (2005), director del Centro de Estudios de Justicia de las Américas [CEJA] y la Corporación Ex- celencia en la Justicia [CEJ] (2011), por tratarse de los textos más completos encontrados. No sobra mencionar que el proceso de búsqueda y vaciado bibliográfico se realizó con el ánimo de hacer un ejercicio descriptivo de los contenidos más relevantes, sin dejar de lado un enfoque crítico a la hora de interpretarlos.

El escrito se divide en cuatro partes. En la primera se expone la discusión conceptual de los modelos de gestión y se aborda a nivel teórico lo que se ha entendido por modelo de gestión en la administración de empresas. En la segunda se demuestra el acuerdo existente sobre la necesidad de crear un nuevo modelo de gestión y de incorporar principios de gerencia, planeación, productividad y liderazgo, propios del mundo empresarial, a la administración judicial. En la tercera se encuadra a Colombia dentro de la discusión y, finalmente, en la cuarta se presentan algunas conclusiones.

\section{DISCUSIÓN CONCEPTUAL DE LOS MODELOS DE GESTIÓN}

Después de revisar la literatura existente a nivel nacional e internacional sobre modelos de gestión de los despachos judiciales, puede decirse que esta, además de no ser muy amplia, se encuentra dispersa y consta, en su gran mayoría, de relatos de experiencias de ciertos países o ciudades que los han implementado en su totalidad o en algunos de sus aspectos específicos (por ejemplo, el uso de TIc, las notificaciones, el reparto, entre otros). En este sentido, se trata de literatura más casuística que técnica o teórica. 
A pesar de ello, resulta indispensable adentrarse en la discusión conceptual de los modelos de gestión para realmente comprender de qué se tratan y cuál es la relación que tienen con el nuevo derecho procesal organizacional. Con esta finalidad, se hace una breve exposición sobre qué se entiende por proceso, por organización y por gestión en la administración de empresas, disciplina del conocimiento en que mayor desarrollo se le ha dado al concepto de modelo de gestión que se ha procurado aplicar en la administración pública, y que se debe empezar a emplear prioritariamente en la administración de los despachos judiciales.

Respecto al concepto de proceso y concretamente de proceso empresarial, las normas estandarizadas Iso $9000^{6}$ han establecido que es el "conjunto de actividades mutuamente relacionadas o que interactúan, las cuales transforman elementos de entrada en resultados" (Organización Internacional de Normalización -ıso, 2005). A su vez, los elementos de entrada son resultados de otros procesos (ISo, 2005, arts. 3.4.1 y 3.4.2). En consecuencia, un proceso empresarial es el conjunto de actividades coordinadas en una estructura organizacional que transforman elementos de entrada en resultados, productos o fines empresariales (Weske, 2012, p. 5).

Ahora bien, un proceso o proceso empresarial no debe confundirse con una organización. Según las precitadas normas estandarizadas Iso 9000,

6 ISO 9000 es una de las normas de gestión de calidad más reconocidas a nivel mundial, que presenta directrices con el propósito de incrementar la eficiencia de un negocio y la satisfacción del cliente. una organización es el "conjunto de personas e instalaciones con una disposición de responsabilidades, autoridades y relaciones" (Iso, 2005, art. 3.3.1). Normalmente estas personas e instalaciones se encargan de realizar procesos. De manera que por gestión se entienden las "actividades coordinadas para dirigir y controlar una organización" (Iso, 2005, art. 3.2.6). Así, en el sentido de la ıso 9000, un sistema o modelo de gestión es el conjunto de elementos que conforman la política, los objetivos y la metodología para lograrlos, la dirección y el control de una organización (Hoyle, 2009, p. 116-117; ıso, 2005, arts. 3.2.1-3.2.3).

Normalmente, se entiende que estos modelos de gestión se concretan en estructuras organizacionales, es decir, en las "formas en las cuales una organización divide sus labores en distintas actividades y luego las coordina" (Hitt, Black y Porter, 2006, p. 230). Según Hitt et al. (2006, p. 240-247), las estructuras organizacionales más comunes son las siguientes: 1.) Estructura por función, que básicamente “ordena la empresa en torno a las áreas de funciones tradicionales como contabilidad, finanzas, marketing, etc." (2006, p. 240), separando el conocimiento especializado de cada área para dirigirlo a la ejecución de procesos de la compañía; 2.) Estructura por producto, en la cual la empresa se organiza en torno a los productos específicos que espera tener como resultado de sus procesos; 3.) Estructura por división, que fracciona la empresa en áreas que agrupan múltiples productos específicos; 4.) Estructura por cliente, que organiza la empresa en torno a las categorías de sus clientes; 5.) Estructura 
por área geográfica/región, en la cual la empresa se divide en áreas geográficas o regiones en las que tiene actividad; 6.) Estructura matricial, donde se superponen dos estructuras organizacionales, haciendo que, por ejemplo, un trabajador tenga que reportar su trabajo a dos jefes de dos estructuras distintas; y 7.) Estructuras organizacionales combinadas, en las que se combinan las diferentes formas de estructuras organizacionales.

Aterrizando los anteriores conceptos a la gestión de la administración de justicia a nivel micro, el despacho judicial, al igual que cualquier organización, requiere de una gestión correcta y técnica para alcanzar los objetivos que se propone (International Consortium for Court Excellence [IFCE], 2013, p. 12; Maan, 2009). Es, justamente, la ausencia de modelos de gestión lo que ha llevado al abismo a los sistemas de justicia de muchos países de la región, ${ }^{7}$ entre ellos el argentino, el costarricense y el peruano, por no hablar únicamente del colombiano, todos transitando como trenes sin carrileras. Por ello se afirma que el derecho procesal se quedó corto a la hora de introducir conocimientos multidisciplinares que permitan administrar una empresa u organización tan grande y compleja como lo es la rama judicial (compuesta en su gran mayoría por despachos judiciales) y, en este sentido, se hace imperativo pensar en un nuevo derecho procesal organizacional.

Para ejemplificar el distanciamiento entre la administración de empresas y el derecho, entre el

7 Para profundizar en estos países, ver bibliografía comentada. entrenamiento en gerencia de despachos y el entrenamiento en lo misional o la ruptura entre lo técnico y lo profesional basta observar lo que sucede actualmente en Colombia y en muchos otros países. En Colombia, la dirección del despacho judicial está a cargo del juez, quien es la autoridad suprema de este y debe cumplir con la función de establecer (i) las directrices generales de funcionamiento y de manejo de su agenda, (ii) definir procedimientos y rutinas de trabajo, (iii) las labores que cumplirá cada funcionario y (iv) cómo se organizará la atención al público, entre otras (Centro de Estudios de Justicia de las Américas [CEJA], s. f.), sin tener los conocimientos específicos para hacerlo. De esta manera, el juez, en vez de estar concentrado únicamente en decidir los casos y en dirigir el proceso, tiene que sacrificar parte considerable del tiempo a resolver cuestiones netamente administrativas.

Los cambios recientes en el sistema judicial (reformas estructurales), el crecimiento del sistema como tal (Rivas Acuña, 2007; Lienhard y Kettinger, 2011) y el aumento de demanda de justicia han motivado fuertes cuestionamientos a la forma como se administra el despacho y han generado alguna bibliografía sobre gestión judicial, ${ }^{8}$ también conocida como individual court management (National Association for Court Management [NACM], 1992) o microgerencia. A esto se suma el generalizado descontento con la calidad, eficiencia, eficacia y valor de la justicia, que también ha promovido reformas en la gestión de los despachos judiciales y ha

8 Ver: Vargas Vianco, J. E. (Septiembre de 2005). 
puesto a pensar a los diseñadores de políticas públicas y a la academia en modelos alternativos de administración judicial (Santos, 2003) o, por qué no, en un nuevo derecho procesal organizacional.

\section{CONVERGENCIAS HACIA UN NUEVO MODELO DE GESTIÓN}

Aunado al aumento de la demanda de justicia y al generalizado descontento con el sistema judicial, el fracaso de las reformas judiciales adelantadas en varios países en años recientes (Hall, Hoffman y Stromsen, 2003) ha hecho concluir a algunos que una buena alternativa es aplicar los conocimientos de la administración de empresas y la gerencia al desarrollo de un nuevo modelo de gestión judicial que impulse la eficiencia del sistema. Lo anterior de ninguna manera implica que en la actualidad no existan pautas de manejo de los despachos judiciales. Sin embargo, es necesario aclarar que se trata de procedimientos empíricos establecidos mediante la costumbre y el afianzamiento de prácticas procesales y no de algo racional, planificado o diseñado específicamente para responder a las necesidades del sistema judicial.

Respecto a la urgencia de pensar en modelos de gestión que atiendan las necesidades contemporáneas de los despachos judiciales ha habido varios pronunciamientos, entre ellos el de Andreas Lienhard y Daniel Kettiger (2011). Según ellos, la única solución para mejorar la eficiencia y producción judicial es trabajar en los modelos de gestión, es decir, en "la actividad administrativa que crea y mantiene los recursos y el personal requerido para poder tomar una decisión judicial” [traducción libre].

Esta posición contrasta con lo que normalmente sucede en algunos países de la región, donde todos los problemas de la justicia se resuelven con reformas procesales, a pesar de que, por ejemplo, en Colombia, las últimas reformas judiciales adelantadas, incluidas las leyes 794 de 2003 y 1395 de 2010, no han alcanzado los resultados esperados y han demostrado ser un fracaso a la hora de resolver los principales problemas de la rama.

La NACM también ha sido un actor importante a la hora de difundir la idea de gerencia judicial y recalcar el tema de los administradores de los despachos judiciales y su necesidad en los juzgados de hoy en día. Según la NACM (1992), Ios despachos judiciales deben tener administradores encargados y algunas funciones que deben desempeñar son: el manejo de los recursos humanos (contratar, seleccionar, entrenar, establecer estándares éticos, aconsejar, administrar el salario, incentivar, etc.), el manejo de la administración fiscal (el presupuesto, su administración, contabilidad, etc.), la distribución de la carga de trabajo, el manejo tecnológico, el manejo de la información, la administración del espacio físico y de la infraestructura, el manejo de la relación con la comunidad, con otras entidades del Estado y con los mismos jueces y el manejo de los servicios secretariales. Ello permitiría al juez concentrarse en la función jurisdiccional y aumentaría el tiempo que puede dedicarle a esta, pues ya no lo desperdiciaría 
decidiendo cuestiones administrativas. Uno de los aspectos que más reitera respecto al administrador es que debe haber constante comunicación entre este y los jueces de los despachos que está administrando para que haya trabajo en equipo y se eviten problemas, y que sus funciones estén claramente establecidas para que no dependa únicamente de las que los jueces le quieran delegar.

A nivel regional, quizá el autor más destacable por su esfuerzo en llamar la atención sobre la necesidad de estudiar y crear modelos de gestión de los despachos judiciales es Juan Enrique Vargas Viancos, director del CEJA. En su texto, Herramientas para el diseño de despachos judiciales (2005), desarrolla varias ideas que vale la pena describir no solo para resaltar la convergencia hacia la importancia del tema sino también para entender en qué estado se encuentra la discusión. Posiblemente, este es el texto que mejor explica la problemática de los modelos de gestión de los despachos judiciales. Lo primero que habría que señalar es la distinción de tres niveles de gestión: el más alto de la organización, el del Gobierno Judicial, que se encarga de la conducción estratégica de la institución; el segundo nivel, la gerencia central del sistema, que responde por la administración a nivel macro; y el tercer nivel, el del despacho judicial, "donde los aspectos de gestión cobran mayor importancia y donde ha sido más difícil introducir mejoras significativas al sistema tradicional de organización y funcionamiento".

Respecto al tercer nivel, Vargas define la gestión como aquello que tiene que ver con la configura- ción de las estructuras institucionales, los procesos de trabajo, la división de roles y la asignación de competencias operativas para que las decisiones jurisdiccionales se generen de la forma más eficaz y eficiente posible. En relación con lo expuesto sobre el fracaso de las reformas procesales, hace explícita la vinculación entre gestión y los modelos procesales, y explica que uno de los principales problemas de la región es la forma desconectada como se deciden y trabajan las reformas sustantivas a la justicia y aquellas referentes a la gestión del sistema. En general, los cambios sustantivos no han producido los efectos que pretendían producir y las reformas a la gestión, al no ser pensadas en función de la prestación del servicio de justicia, han legitimado y profundizado formas de trabajo disfuncionales o han retardado los cambios de fondo que se requieren. Por esto, en su opinión, resulta esencial considerar debidamente la forma como se relaciona la concepción del trabajo sustantivo de la institución y los mecanismos de gestión de este para evitar modelos de administración incompatibles con los modelos procesales. Las ideas de este autor son un sustento más de la necesidad de pensar en la creación de un nuevo derecho procesal organizacional que se encargue del estudio de los modelos de gestión y de su compatibilidad con los modelos procesales.

Respecto a la relación entre modelo de gestión y modelo procesal, según Vargas (2005), debe tenerse en cuenta que en los procedimientos escritos la labor principal del despacho es la construcción del expediente para que, una vez 
acumulada toda la información en él, el juez pueda dictar una sentencia. Debido a esto, intervienen un número elevado de empleados judiciales que colaboran en la formación del expediente, ya sea elaborando borradores de resoluciones, tomando declaraciones o recibiendo pruebas. Por su parte, en los procedimientos orales las decisiones son tomadas directamente por los jueces en audiencias, por lo que los recursos del despacho deben dirigirse a la más eficiente organización de estas. Adicionalmente, el recurso juez pasa a ser más significativo y los empleados judiciales ya no necesitan tener una formación jurídica para desempeñar sus funciones.

Vargas (2005) menciona que existen algunos principios orientadores para lograr un funcionamiento más eficiente y que quizá reúnen la mayoría de las propuestas que se han hecho al respecto:

i) Diferenciar las funciones jurisdiccionales de las administrativas.

ii) Aprovechar al máximo las economías de escala, introduciendo unidades jurisdiccionales grandes para facilitar las labores administrativas y evitar duplicar esfuerzos.

iii) Tender hacia la profesionalización de la administración, ya que los jueces no están preparados ni tienen el conocimiento requerido para gestionar un despacho judicial. Por ello, es importante incorporar administradores con poder propio y real. iv) Privilegiar los diseños simples y flexibles que hagan más énfasis en los resultados que en los procedimientos.

v) Contar en todo momento con información oportuna y de calidad para tomar las mejores decisiones en materia de gestión.

Sobre el último principio orientador, hace énfasis en la importancia de tener información confiable para poder escoger un modelo de gestión que sea apto para las necesidades de cada país y de cada juzgado. Es fundamental, continúa diciendo, tener información sobre los ingresos esperados para poder distribuir los recursos del sistema en la forma más racional; saber el número y tipo de casos que ingresan en determinados lapsos de tiempo, los factores que pueden influir en ese volumen esperado, las características de los usuarios, la duración posible de los litigios y el índice probable de apelabilidad, entre otros; y pensar en la posibilidad de abandonar la unidad de medida de la carga de trabajo tradicionalmente utilizada (la causa, la denuncia o el expediente judicial) debido a que muchas veces esta no transmite exactamente la carga de trabajo. En otras palabras, una misma causa puede involucrar a muchas personas, puede ser un asunto simple o complejo, etc.

Simultáneamente a determinar la demanda del sistema, menciona que también es importante analizar la oferta o los recursos con que se cuenta. Deben conocerse los recursos humanos disponibles, su nivel de capacitación, y los recursos materiales (infraestructura judicial y su situación jurídica, recursos informáticos, recur- 
sos presupuestarios). Igualmente importante para él es analizar las fortalezas y debilidades del sistema de gobierno judicial, ya que de éste depende la capacidad de dirigir adecuadamente el proceso de cambio.

Uno de los aspectos más importantes del texto de Vargas es lo referente a la estructura de las oficinas o despachos judiciales. La organización tradicional de los juzgados en América Latina, incluida Colombia, está compuesta por un juez, un secretario y un conjunto de empleados que cooperan en su trabajo en un mismo espacio físico. Cada juez trabaja de forma desconectada de sus colegas y replican todas las funciones que requieren para cumplir con su cometido. Por ello, afirma, este tipo de organización es poco eficiente y hace muy costoso ampliar la cobertura judicial, pues siempre es necesario crear un despacho completo. Además de estas desventajas, esta forma de organización genera muchas dificultades para establecer sistemas equitativos de repartición de la carga de trabajo.

Por lo anterior, propone algunas alternativas de estructura institucional como, por ejemplo, los servicios comunes, a los que define como "unidades que realizan sus funciones no sólo para un juzgado determinado sino para un conjunto de ellos, generalmente con cercanía física" (Vargas, 2005, p. 88) y van desde la administración física de los inmuebles donde funcionan los juzgados hasta funciones típicamente ligadas al trabajo jurisdiccional, como son las notificaciones, atención al público, sistemas de información, etc. Además de los servicios comunes, menciona una estructura institucional aún más eficiente, en la que un conjunto de jueces, bajo un esquema de trabajo habitual comparten una secretaría y a pesar de estar agrupados, no pierden el carácter de unipersonales, pues aún siguen tomando sus decisiones individualmente. Este tipo de modelo permite que no solo los servicios comunes pasen a ser brindados centralizadamente, sino también todas las labores de apoyo.

Al igual que la NACM (1992), Vargas coincide en que existe una tendencia actual, consistente en “la contratación de administradores para ejecutar las tareas de gestión en los tribunales [juzgados] pluripersonales de dimensiones suficientemente grandes como para que sea abordable económicamente la inclusión de estos profesionales" (2005, p. 96). Estos administradores, dice, deben cumplir varias funciones entre las que se encuentran: i) entregar los insumos técnicos para la adopción de las decisiones generales sobre la gestión, por ejemplo, sobre los criterios para la contratación y control del personal, para la distribución de las causas entre los jueces, las inversiones, las adquisiciones, etc.; ii) manejar el personal de los juzgados, es decir, asignarles el trabajo y controlar su ejecución; iii) manejar la agenda y iv) servir de comunicadores con la gerencia central en los temas vinculados a la gestión.

En relación con la segunda función, menciona que lo ideal es que el personal no esté adscrito en forma directa a cada juez sino a un conjunto de ellos, aunque aclara que esto es difícil de aceptar por los jueces, pues están acostumbrados a nombrar su propio personal y a establecer 
las rutinas de trabajo que consideran más convenientes, lo que termina generando muchas formas dispares de funcionamiento y muchas ineficiencias. Adicionalmente, esta forma de administración de personal únicamente es posible cuando hay una separación tajante entre los roles jurisdiccionales y los administrativos (Vargas, 2005).

Otro aspecto que considera clave dentro del modelo de gestión es el manejo de la agenda del juzgado por parte de los administradores. Normalmente los jueces se reservan celosamente esta parte para ellos ya que están acostumbrados a trabajar a su propio ritmo y no son muy dados a que otros dispongan de su tiempo. Lo ideal sería que estuviera en cabeza de los administradores, si se tiene en cuenta que el tiempo es el recurso más valioso y debe sacársele el mayor provecho y rendimiento.

Pastor Santos (2003), en una revisión de los cambios acaecidos en América Latina en los procesos de reforma judicial relacionados con los sistemas de organización y gestión o gerencia de la administración de justicia en los últimos años, al igual que Vargas Viancos (2005), señala una tendencia hacia la especialización de los jueces, y la concentración de estos para hacer uso de las economías de escala, el desarrollo de los servicios comunes, el nombramiento de administradores en los despachos judiciales, la implementación y optimización de los recursos informáticos, el avance en materia de estadística judicial y el mayor énfasis en eficiencia con calidad.
Al igual que se ha advertido en otras latitudes, en Argentina, Garavano et al. (1999) sostienen que el fracaso de las reformas judiciales en su país se debe a que "dejan intacta la organización burocrática encargada de llevar adelante las tareas jurisdiccionales, esto es, la Oficina Judicial", olvidando que es el soporte del órgano jurisdiccional al ser una típica oficina administrativa y que su principal problema es que padece de un exceso de trámites. Por ello, los nuevos códigos expedidos, el aumento de los juzgados con más personal y la dotación de soportes tecnológicos no implican en sí mismos mayor calidad o menor retraso. Según ellos, debe rediseñarse el flujo de trabajo interno y redefinirse los roles y funciones, para lo cual proponen crear oficinas de apoyo administrativo o secretarías comunes, dividir la carga de trabajo y distinguir, de entrada, los casos sencillos de los complejos para sacar provecho a la división de trabajo.

Ahora bien, a pesar de la convergencia señalada respecto a la necesidad de diseñar modelos de gestión para los despachos judiciales, esta idea también ha tenido que enfrentar algunos opositores. Uno de los argumentos en los que más se ha insistido a la hora de oponerse a la gestión o administración judicial es la vulneración de la independencia y autonomía de los jueces. Sobre la tensión existente entre la independencia judicial y los modelos de gestión, Bert Maan (2009), juez holandés, examina los problemas que podrían surgir frente a una gestión judicial basada en limitaciones presupuestarias, y recalca la necesidad de ver los despachos judiciales como organizaciones que deben ser dirigidas 
por profesionales o administradores para poder cumplir los objetivos que se proponen y satisfacer las necesidades de la comunidad.

Asimismo, la mayoría de los jueces también se han mostrado reacios a la introducción de la microgerencia. Vargas (2005) indica que una de las lecciones que ha aprendido es que, paradójicamente, "Ios jueces tienden a resistir mucho más vehemente cambios organizacionales y de gestión que las reformas sustantivas aun cuando estas sean de gran envergadura" situación que para él está relacionada con el hecho de que los jueces han hecho recaer su poder, más en sus facultades administrativas (la posibilidad de designar empleados, de asignar carga de trabajo y controlar su ejecución, de disponer el destino de ciertos recursos, etc.) que en sus potestades jurisdiccionales.

Desde una perspectiva más constitucional, la propuesta de concentrar a los jueces para poder hacer uso de las economías de escala también ha recibido críticas. De acuerdo con sus detractores, no es posible concentrar y distanciar demasiado los juzgados de la población pues ello vulneraría el derecho fundamental al acceso a la justicia (Vargas, 2005). Aun así, dentro de la escasa y dispersa literatura parece haber más acuerdo que desacuerdo sobre la necesidad de acercar la gestión y las ciencias de administración a los despachos judiciales. Si bien existen reparos como los acabados de mencionar, ningún texto ni persona defiende que los modelos de gestión de los despachos judiciales no son un asunto indispensable y un tema pendiente de la justicia. Así entonces, lo que resta es po- pularizar el concepto y hacer de él una prioridad en la agenda nacional.

\section{COLOMBIA EN EL DEBATE}

Si en términos generales el debate sobre la gerencia judicial es bastante reciente, en Colombia se trata de un asunto aún más novedoso que apenas comienza a despegar. No obstante lo anterior, la $\mathrm{CE}^{9}{ }^{9}$ ha liderado en el país los debates en torno a los modelos de gestión y ha intentado impulsar en el Consejo Superior de la Judicatura (csJ) reformas en esta materia. En el 2008, adelantó el proyecto Propuesta de ajuste al modelo de gestión de los juzgados civiles municipales de Bogotá, D. C. que culminó con un informe publicado en junio de 2011. Debido a que es, quizás, lo mejor que se ha escrito a nivel nacional, a continuación se describen algunas de las propuestas plasmadas en el informe para visualizar en qué nivel se encuentra la discusión en Colombia.

Lo primero que debe destacarse es que en la presentación del informe, la CEJ (2011) reconoce que, a partir de diferentes experiencias nacionales e internacionales, los modelos de gestión podrían categorizarse en tres: i) aquellos que minimizan la carga administrativa en los jueces, asignándola a equipos dedicados a este fin; ii) los que a esta experiencia agregan la separación de funciones procesales en las de mero trámite y las sustantivas, dejando las primeras a cargo

9 Organización no gubernamental del sector privado que busca contribuir al mejoramiento de la administración de justicia de Colombia y hace control ciudadano a los administradores de la rama judicial. 
de equipos de trabajo de naturaleza secretarial, pero con capacidad de decisión sobre los asuntos atribuidos; y iii) los que dejan estas competencias o algunas de ellas a cargo de entidades de orden administrativo e incluso privado.

Además de esto, advierte que en la actualidad los juzgados civiles municipales cuentan con un modelo de gestión definido y puesto en funcionamiento por el css para el modelo procesal escrito. Lo anterior va en contravía de la construcción de un modelo procesal oral que actualmente está siendo implementado en el país. A pesar de esto, "el éxito o el fracaso de un modelo de gestión no es atribuible únicamente a variables endógenas a la Rama Judicial, sino a condicionamientos de orden normativo (competencia del juez, normas procesales, etc.), de política económica y social (recesión, tasas de interés, desempleo, etc.) o presupuestal" (CEJ, 2011, p. 6).

Al igual que Vargas Viancos (2005), la ces (2011) también reconoce los distintos niveles de gestión: gobierno, gerencia y operación de la unidad del despacho judicial. En Colombia estos tres niveles estarían representados por el Consejo Superior de la Judicatura (gobierno), la Dirección Ejecutiva de Administración Judicial, caso particular de los juzgados civiles municipales de Bogotá (gerencia) y los despachos de los jueces (operación).

En cuanto a las propuestas de ajuste a los modelos de gestión que hizo la cEJ, el informe aclara que se hicieron teniendo en cuenta la efectividad material de las decisiones judiciales, la separación efectiva de las funciones administrativas y jurisdiccionales, la profesionalización de los servicios administrativos, el aprovechamiento de economías de escala y tecnologías de información y comunicación y la capacidad de gestión de todas las dependencias involucradas, en especial su capacidad para administrar y solventar los flujos y cargas de trabajo. Con base en estos principios orientadores las propuestas que plantea son:

i) Especializar los juzgados civiles municipales por competencia material.

ii) Separar efectivamente las funciones administrativas de las jurisdiccionales.

iii) Depurar el inventario de los procesos a cargo de los jueces civiles municipales.

iv) Fortalecer el desarrollo de tIC para modernizar la justicia civil municipal.

v) Mejorar la atención al usuario de la justicia.

vi) Mejorar la calidad de los servicios a cargo de los auxiliares de la justicia.

vii) Fomentar la disciplina procesal en los procesos civiles.

Antes de pasar a explicarlas brevemente es necesario recalcar que estas propuestas fueron planteadas únicamente para los juzgados civiles municipales de Bogotá y no necesariamente son aptas para otras jurisdicciones, debido a que las necesidades son distintas. Igualmente, es relevante señalar la consonancia de estas 
propuestas con las de Vargas Viancos (2005), lo que permite identificar una tendencia regional en cuanto a lo que se considera ideal para los modelos de gestión.

La primera propuesta de la CEJ plantea desagregar la carga de los juzgados civiles municipales de la ciudad para que el trámite de las causas corresponda a diferentes tipos de juzgados en el mismo nivel jerárquico. Para ello propone subdividir la ciudad en dos circuitos y crear cinco tipos de juzgados: (i) jueces desconcentrados multicompetencia para el conocimiento de las pequeñas causas civiles, (ii) jueces concentrados de sustanciación civil, (iii) jueces concentrados de trámite, (iv) jueces concentrados especializados en ejecución civil y (v) jueces civiles de conocimiento ordinario para el trámite de las pretensiones declarativas de menor cuantía y otras. Esta propuesta también incluye establecer una oficina de ejecuciones civiles para soportar la labor de cobro, entre cuyas funciones valdría la pena mencionar la localización de bienes para embargo y secuestro, la realización del remate, la liquidación de créditos y costas, entre otras. Este esquema funcional de la justicia civil permitiría que el mayor volumen de procesos, los de ejecución civil, puedan ser tramitados por jueces especializados.

La segunda propuesta, concerniente a la separación efectiva de las funciones administrativas de las jurisdiccionales, propende por fortalecer las actividades de apoyo administrativo mediante un centro de servicios independiente de los despachos judiciales, que debe encargarse de la radicación, reparto de demandas y memo- riales, comisiones judiciales, pruebas anticipadas, notificaciones de todo tipo, orientación al usuario, designación y control de la actividad de auxiliares de la justicia, gestión integral del archivo documental, administración de la información estadística, manejo de los depósitos y títulos judiciales y apoyo informático y coordinación de salas de audiencia. Esta propuesta incluye la creación de puntos desconcentrados de atención a usuarios, en los que se permita la radicación de demandas y otros memoriales y se brinde orientación.

La tercera propuesta tiene que ver con la depuración del inventario de los procesos a cargo de los jueces civiles municipales, debido a que el inventario inactivo afecta el análisis de carga efectiva en la medida en que infla la percepción de congestión judicial y, por esta razón, es preciso avanzar en el archivo definitivo de los procesos inactivos. Señala además la cEJ que las medidas de perención, desistimiento tácito y el fortalecimiento de las salidas tempranas (sentencias anticipadas, conciliaciones, etc.) son estrategias que ayudarán a controlar la carga efectiva.

La cuarta propuesta está relacionada con el fortalecimiento del desarrollo de Tıc para modernizar la justicia civil municipal. Los motivos detrás de esta propuesta tienen que ver con los beneficios de la introducción de las TIC al sistema judicial, pues constituyen mecanismos destinados a dotarlo de eficiencia, eficacia, equidad, transparencia, acceso a la justicia, gobernabilidad y protección de derecho. En este sentido, la CEJ (2011) propone desarrollar un sistema de ges- 
tión procesal o de administración de casos que supere las limitaciones del actual siglo XXI, dotarlo de hardware de importante capacidad para que la velocidad no continúe siendo una barrera para la alimentación de éste, formar a los jueces y empleados judiciales en su uso, incluir en la página web de la rama judicial la información útil en la orientación del ciudadano y maximizar la utilización de la intranet para las comunicaciones entre jueces y entre estos y el centro de servicios administrativos. Esta propuesta debe tender hacia la gradual implementación del expediente virtual y el litigio en línea.

La quinta propuesta CEJ (2011) es mejorar la atención a los usuarios garantizando la concreción del derecho en comento y ofreciendo pronta y cumplida justicia. Para ello es importante que estos conozcan la oferta judicial para la tramitación de sus conflictos, las particularidades bajo las cuales su pretensión será sometida a discusión, los costos, los tiempos, las formas alternativas para resolver su conflicto, etc. Para ello propone una oficina de orientación al ciudadano en el centro de servicios administrativos, la atención telefónica mediante una línea 1-8000 y la implementación en la página web de un link que permita a los usuarios comunicarse en línea, así como la divulgación de información estadística, normativa y jurisprudencial.

La sexta propuesta se refiere al mejoramiento de la calidad de los servicios a cargo de los auxiliares de la justicia, debido a que los diagnósticos sobre la congestión judicial han evidenciado la dilación procesal generada por diferentes factores que giran alrededor de su designación, posesión y desempeño de la labor a ellos asignada. Por ello, la ces (2011) propone redefinir los criterios de selección de estos funcionarios y el aumento de los requisitos a efectos de profesionalizar el servicio, construir una nueva lista de auxiliares, modificar los parámetros para la designación y control de desempeño, viabilizar el ejercicio de la competencia disciplinaria sobre ellos, someter la inscripción al pago de una póliza anual de responsabilidad civil para amparar los riesgos de una mala gestión, establecer limitaciones para la inscripción en más de dos oficios y la asignación de más de tres actividades de apoyo judicial al mismo tiempo, uniformizar las remuneraciones y designar y monitorear la actividad a través de los centros de servicios.

La séptima y última propuesta concierne al fomento de la disciplina procesal en los procesos civiles para evitar que las partes de un proceso actúen dilatoria y abusivamente ocasionando mora procesal, como suele suceder en Colombia. Para ello, dice la CEJ, es necesario generar un cambio cultural que permita al juez asumir con responsabilidad su rol de director del proceso y a los intervinientes las suyas. En este orden de ideas, propone reformas normativas que permitan al juez sancionar con severidad las actuaciones dilatorias y encarecedoras del proceso, dotarlo de la potestad para fijar el plan de caso (programar junto con las partes las audiencias y diligencias del proceso), permitir el cobro anticipado de las expensas judiciales, entre otras.

En resumidas cuentas, podría decirse que en Colombia el debate sobre los modelos de gestión se encuentra en un nivel teórico y propen- 
de por el modelo y las propuestas elaboradas por la Corporación Excelencia en la Justicia, es decir, por la creación o implementación de servicios comunes, la separación de funciones administrativas y jurisdiccionales y el aumento en la utilización de las Tıc, en términos generales.

Por otro lado, está la experiencia que da a conocer Lucía Arbeláez de Tobón (1996) sobre el modelo de despacho judicial implementado en los juzgados de Itagüí y de Envigado ${ }^{10}$ como una solución alternativa para los problemas que enfrentaban. Estos proyectos fueron fruto de una iniciativa público-privada que contó con el apoyo de varias corporaciones, entre ellas la ces.

La idea principal fue incorporar a los despachos judiciales principios de gerencia, planeación, productividad y liderazgo, propios del mundo empresarial, pero extraños y desconocidos en la administración pública y judicial. El primer paso fue la introducción de la administración conjunta de varios despachos judiciales que funcionaban en un mismo municipio, a través de la creación de una secretaría común Ilamada Oficina de Apoyo Judicial, actualmente denominada Centro de Servicios Administrativos. En otras palabras, se pasó del despacho judicial tradicional (cultura de trabajo individualista) a uno que optimizó tiempos y procesos por medio del trabajo en equipo, la organización administrativa y la sistematización, automatización y redistribución de las funciones; la actividad del juez se concentró

10 El 23 de agosto de 2013 visité los juzgados de Itagüí y de Envigado y me reuní con varios jueces, quienes me explicaron cómo fue todo el proceso de creación y de implementación del modelo, así como sus ventajas y retos. en las funciones jurisdiccionales, dejando en la secretaría común el contacto con el público, la recepción de documentos, información, reparto, notificaciones, estadística, entre otras tareas.

El modelo partió del supuesto de que las labores administrativas son meramente instrumentales, por lo que debía liberarse de esa carga al juez y proceder a su ordenamiento, estandarización y normalización para hacer de ellas una rutina de fácil ejecución que sirviera de punto de apoyo a las tareas jurisdiccionales.

Un aspecto interesante de este proyecto fue la etapa de educación legal a la comunidad, en la cual los jueces se proyectaron a la ciudadanía mediante un ejercicio social educador, formador y orientador, a través de publicaciones de interés general (tutela, matrimonio civil, etc.); conferencias sobre distintos aspectos con empresarios, funcionarios públicos, líderes, jóvenes, etc.; un programa radial y un video institucional, entre otros. Hoy en día los funcionarios de esos despachos aseguran que gracias a estas actividades recuperaron la legitimidad de la institución, y que el control ciudadano es fundamental para el mantenimiento de la calidad del sistema, pues los reclamos son una oportunidad para mejorar (reunión personal, 2013).

Los resultados de esta experiencia han sido más que positivos. De acuerdo con las evaluaciones de la rama judicial, mientras el grado de conocimiento en temas administrativos y de gestión era de 3.7 en los juzgados de Itagüí, en los juzgados tradicionales era de 2.5; respecto a la cultura organizacional, los juzgados de 
Itagüí obtuvieron el puntaje de 4.4 mientras los juzgados tradicionales sólo llegaron al 3.2; en clima organizacional, los juzgados de Itagüí registraron 4.0 mientras los tradicionales 3.5. Adicionalmente, el inventario de procesos en esos juzgados se redujo sustancialmente (Arbeláez de Tobón, 1996). Por todo lo anterior, el modelo mostró ser eficiente para la descongestión a pesar de que los ingresos de procesos han aumentado en los últimos años. También convirtió a los jueces en gerentes de sus despachos judiciales, humanizó la justicia, mejoró la atención al usuario, mejoró la imagen de la administración de justicia ante la ciudadanía y recuperó la confianza y la credibilidad en la justicia (Arbeláez de Tobón, 1996). Al decir de Diego Estrada Giraldo (2013), juez representante del sistema integral de gestión y juez civil del circuito de Itagüí, "el sistema de gestión y calidad legitimó y empoderó a la justicia". Sobre las claves del nuevo modelo de gestión, éste aseguró: el trabajo en equipo, el enfoque en la atención al usuario y la comunicación interna eficaz, aspectos que resaltan la importancia de un enfoque sistémico a partir de unas funciones engranadas que producen un resultado.

Por último, en el ámbito nacional hay que mencionar al cs,, corporación que en el documento Modernización de los modelos de gestión en los despachos judiciales de la especialidad civil (s. f.) comenzó a hablar sobre modelos de gestión. En el Plan Sectorial de la Rama Judicial para el cuatrienio 2003-2006, el CSJ (2010) plasmó el propósito de incluir políticas para mejorar la eficiencia, eficacia, calidad y transparencia de la administración de justicia a través del for- talecimiento de los modelos de gestión de los despachos judiciales, entre otros mecanismos. Esta propuesta surgió del Proyecto para el Mejoramiento en la Resolución de Conflictos Judiciales, que inició en el 2000 la Sala Administrativa del css con el apoyo financiero del Banco Mundial, con el propósito de comprobar si un cambio en la organización integral al interior de los juzgados civiles del circuito lograba mayor agilidad en las decisiones sometidas a su juicio e incrementaba la productividad. Las conclusiones de este proyecto también fueron bastante favorables ya que demostraron que el índice de resolución y el índice de evacuación aumentaron y disminuyó el índice de congestión. Estos logros fueron el resultado de disminuir el formalismo en los procedimientos, racionalizar la intervención procesal de las partes, optimizar las prácticas laborales al interior del juzgado mediante la definición de tareas y la colaboración armónica en su ejecución, adecuar la infraestructura física y organizacional, modernizar la tecnología al servicio de los despachos judiciales, adoptar un modelo de archivo y manejo de documentos y crear sistemas y espacios de información actualizada sobre los asuntos de competencia de los juzgados.

En el Plan Sectorial de Desarrollo de la Rama judicial para el cuatrienio 2011-2014 (CSJ, 2010) la eficiencia pasó a ocupar un lugar más importante o, al menos, así lo quiso mostrar el csı. De hecho, el plan se titula Hacia una justicia eficiente, un propósito nacional. No obstante lo anterior, su lectura minuciosa evidencia que, en realidad, la entidad encargada de administrar la rama no se enfocó en la gestión y gerencia judi- 
cial sino que continuó inmiscuida en el error de pensar que las reformas procesales son soluciones a la congestión judicial. Refiriéndose a los retos para el nuevo cuatrienio, el css (2010) menciona que uno de ellos es "garantizar la eficiencia de la administración de justicia con el establecimiento de nuevos procesos y procedimientos en el trámite con tecnologías y adecuadas arquitecturas para la prestación del servicio que disminuyan el tiempo de respuesta”. ¿Nuevos procesos y procedimientos? ¿Acaso no es eso lo que llevamos haciendo los últimos años en Colombia sin obtener los resultados deseados? Resulta notorio el atraso del csı a pesar de que ha comenzado a hablar de modelos de gestión, aunque con un enfoque errado. Esta entidad insiste en medidas de corto plazo, tales como nombramiento de jueces de descongestión o acortamientos procesales, que de ninguna manera evitan modalidades de trabajo ineficientes, sino que por el contrario las reproducen.

Sin embargo, con la aprobación del nuevo Código General del Proceso (cGP) que ordenó a la Sala Administrativa del csı realizar, dentro de los seis (6) meses siguientes a su promulgación, un Plan de Acción para la implementación de dicho cuerpo normativo que incluyera, entre otros, un nuevo modelo de gestión, estructura interna y funcionamiento de los despachos y dependencias de apoyo, el csı pareció haberse relacionado un poco más con las tendencias actuales sobre gestión judicial. Al efecto, conformó una comisión compuesta por algunos jueces y magistrados de la República, quienes presentaron el proyecto final que fue aprobado mediante el Acuerdo PSAA13-9810, del 11 de enero de 2013.
En el acápite Nuevo modelo de gestión y estructura interna de despachos, oficinas y centros de servicios administrativos, reconoce que la necesidad de un nuevo modelo de gestión se debe a la implementación de la oralidad en los procesos, circunstancia que requiere un plan de modernización de la justicia para aprovechar las tecnologías de la información y comunicación. Con esto debe resaltarse el esfuerzo de articular el modelo procesal con el modelo de gestión, algo nunca antes visto en Colombia.

El Plan de Acción sugiere las siguientes medidas para adoptar un nuevo modelo de gestión: i) fortalecer el papel del juez como director del proceso judicial, liberándolo de las funciones que no sean inherentes a su cargo y ii) establecer estructuras tales como las oficinas y centros de servicios administrativos para que asuman las labores administrativas y secretariales a cargo de los despachos judiciales. Para lograr lo anterior propone: i) identificar labores administrativas que están a cargo de los despachos judiciales para ser trasladadas a las dependencias de apoyo; ii) definir la planta de personal de las dependencias de apoyo para que puedan asumir las labores administrativas y secretariales a cargo de los despachos; iii) diseñar protocolos de atención a los usuarios y abogados; iv) adecuar físicamente las dependencias de apoyo; v) redefinir el papel del juez a la luz de las introducciones del cGP; vi) redefinir la planta tipo de los despachos (juez, abogado asistente, sustanciador, auxiliar técnico y escribiente); vii) crear salas de decisiones fijas en los Tribunales Superiores de Distrito; viii) diseñar e implementar mapas de procesos y procedimientos judicia- 
les; ix) diseñar e implementar protocolos de audiencia; $x$ ) implementar un sistema de gestión de calidad; xi) adecuar la infraestructura física y tecnológica para la realización de audiencias orales; xii) incorporar las TIC en diferentes actividades judiciales; xiii) aumentar la cobertura de los sistemas de información y adecuación de los mismos para la implementación del proceso oral y digital; xiv) diseñar e implementar el Plan de Sistema Justicia Digital; xv) diseñar e implementar un sistema de gestión documental; xvi) suministrar bibliotecas en línea para los despachos judiciales; xvii) determinar las cargas razonables de trabajo; xviii) diseñar un modelo de medición de la gestión; xix) formular un sistema de calificación; $x x$ ) implementar un modelo de gestión para el proceso digital; $x x i)$ definir la planta tipo de las Oficinas de Ejecución de Sentencias Civiles (csJ, 2012).

Así las cosas, el csı parece haberse encaminado en la dirección correcta, aunque estamos hablando de un Plan de Acción elaborado recientemente y que aún no puede mostrar sus frutos. Sin embargo, el solo hecho de que el csı esté hablando de implementar un sistema de gestión de calidad, un sistema de gestión documental y un sistema de calificación es un avance en sí mismo, si consideramos que hasta la fecha esta entidad era ajena a estos temas. Aun así, la entrada en vigencia del cGP, que estaba prevista para el $1^{\circ}$ de enero de 2014 , ha generado y continúa generando muchas incertidumbres, pues a pesar de existir el Plan de Acción, este aún no se ha implementado, lo que de ninguna manera permite hacer el cambio radical a la oralidad. Por esta razón, a pesar de considerar que el
Plan de Acción representa un avance, aún falta mucho camino que recorrer.

Con esto en mente, podría decirse que la discusión a nivel nacional se ha acercado un poco a la regional y que Colombia está empezando a entender la importancia de la gestión judicial, a juzgar por las propuestas que han comenzado a surgir para el mejoramiento de la eficiencia de la justicia. Las más populares tienen que ver con la separación de funciones, mayor implementación de las TIC, ${ }^{11}$ aprovechamiento de secretarías comunes, capacitación y mejor atención al usuario. El reto, entonces, es implementar estas propuestas y hacer de la gerencia judicial un asunto de interés nacional para que haya mayor conocimiento sobre los procesos y actividades de esta rama del poder público.

\section{CONCLUSIONES}

A raíz de la revisión crítica de la literatura sobre modelos de gestión pueden deducirse las siguientes conclusiones que, a manera de aporte, pretenden impulsar el desarrollo de la discusión sobre esta materia:

\section{A. La necesidad de crear una base de conocimien- to y de introducir la gestión del conocimiento en la gerencia judicial}

Tradicionalmente, las organizaciones, entre ellas la rama judicial, han gestionado el conocimiento de manera intuitiva y no deliberada. Sin

11 Respecto al uso de las tecnologías de la información y la comunicación en el proceso judicial ver: Londoño Sepúlveda, N. R. (Enero-junio 2010). 
embargo, durante la última década ha habido un creciente reconocimiento de que una gestión más racional y activa del conocimiento puede aportar importantes beneficios a las organizaciones. Entre estos beneficios se encuentra la conversión de ideas en capital, la transformación de conocimiento individual en conocimiento colectivo y el mejoramiento del flujo del conocimiento (Ministerio de Justicia de Nueva Zelanda, s. f.)

En este contexto, uno de los principales hallazgos de esta investigación es que no existe gestión del conocimiento en esta materia; la información sobre gerencia judicial se encuentra bastante fragmentada, dispersa; y no existe univocidad en la forma como se utiliza el término modelos de gestión. A ello se suma la escasez de información completa y relevante. Como consecuencia de esto, el aprendizaje del tema se dificulta, se obstaculiza su difusión y se distorsiona el concepto.

Así, los vocablos sistemas de gestión, gerencia y reorganización han sido utilizados para referirse a varias cosas (Pandey, 2011), entre ellas:

i) procesos de concentración espacial, esto es, reunión de órganos judiciales dispersos en un único centro o en unos cuantos, reduciendo así el grado de atomización territorial; ii) la incorporación de nuevas tecnologías en la administración de justicia que permiten desde la simple informatización de la tramitación del proceso hasta la creación de sistemas informáticos para la gestión procesal (SIGP) y que favorecen la agilización de las comunicaciones mediante el correo electrónico o que facilitan a las partes el acceso a la información sobre la marcha del proceso; iii) la incorporación de criterios y pautas de mejora de la eficiencia mediante la utilización de criterios propios de la gestión pública (redefinición de los despachos y los puestos de trabajo, incorporación de profesionales de la gestión, fortalecimiento de los mecanismos de control interno); iv) el aprovechamiento de los sistemas de información, tanto de las estadísticas judiciales como de los sistemas informáticos de gestión procesal; v) la rendición de cuentas; vi) la formación de jueces y de personal no judicial, entre otras" (Santos, 2003).

Por esta razón, resulta indispensable generar información de buena calidad, compendiar la literatura que ya existe y crear una base de conocimiento para llamar la atención sobre la importancia de la gerencia judicial y de modelos de gestión que permitan llevar a cabo una adecuada y eficiente administración en el sector. Un primer resultado sería el almacenamiento y sistematización de conceptos, experiencias y datos para ser consultados y utilizados en aras del mejoramiento de la administración de justicia.

\section{B. La verdadera finalidad de la gerencia judicial y} la necesidad de incluir a los funcionarios judiciales en el debate

Uno de los grupos más resistentes a la introducción de modelos de gestión y gerencia judicial en los despachos judiciales han sido los mismos jueces y los funcionarios judiciales de los juzgados. Esta obstinación e intransigencia proviene de la noción equivocada de que la gestión judicial acabará con la independencia de los jueces y terminará privatizando la justicia, 
industrializándola e introduciendo una especie de capitalismo o fordismo judicial, en donde los funcionarios pasarían a ser meros instrumentos de la cadena de producción. Desconoce este imaginario desafortunado, que detrás de la incorporación de principios de gerencia, planeación, productividad y liderazgo, propios del mundo empresarial, a la administración de justicia, está el propósito de aumentar la calidad de vida de los trabajadores judiciales, respetar los derechos de la ciudadanía a una pronta y cumplida justicia y propender por el cumplimiento de una de las mayores funciones del Estado: la función jurisdiccional.

En este orden de ideas, resulta imperativo incluir a los jueces y a los funcionarios judiciales en el debate, ya que son ellos los que mejor conocen el sistema y, como consecuencia de ello, constituyen una gran fuente de innovación. Adicionalmente, su participación en el diseño del nuevo modelo de gestión es indispensable por ser quienes primero harán uso de éste y quienes deberán apropiarse de él y empoderarse mediante él. Esto explica, en gran medida, el éxito de las experiencias de Itagüí y Envigado.

\section{La insuficiencia del derecho procesal formal y la necesidad de crear un derecho procesal orga- nizacional}

La tercera conclusión está relacionada con el hecho de que el derecho procesal no agota el estudio de los modelos de gestión, tal y como se mencionó en la introducción. De acuerdo con esta postura sostenida a través del texto, el derecho procesal se quedó corto a la hora de incluir en su objeto de estudio una de las variables más importantes para la realización del derecho objetivo, a saber, la gerencia judicial y los modelos de gestión. Ello, debido a que no es posible pensar que únicamente con normas procedimentales se garantizará el derecho al acceso a la justicia. Quizá un ejemplo retrate mejor la problemática: sería absurdo creer en la garantía del derecho a la libertad de locomoción únicamente con la expedición del Código Nacional de Tránsito y sin una infraestructura vial que permita su efectiva realización. De la misma manera, no parece lógico que con simples códigos de procedimiento, que nada dicen respecto de los modelos de gestión, se obtendrá una verdadera justicia material. Por ello, es inaplazable avanzar en la creación de un nuevo derecho procesal organizacional, cuyo estudio esté concentrado en la gerencia judicial y en los modelos de gestión de los despachos judiciales como mecanismos para garantizar el derecho subjetivo.

\section{La necesidad de agilizar el debate en Colombia}

Respecto a la discusión adelantada en Colombia, debe advertirse que la calidad del debate ha sido en su gran mayoría pobre y su avance muy lento. Sólo hasta la expedición del Plan de Acción para la Implementación del Código General del Proceso, en diciembre de 2012, la entidad pública encargada de la administración de la rama judicial, el csı, enfocó su discurso en los modelos de gestión de los despachos judiciales. Antes de eso, todas las reformas procesales fueron pensadas y aprobadas sin tener en cuenta el modelo de gestión ni la infraestructura judicial 
necesaria para introducir los cambios. Muestra de lo anterior es la aprobación del nuevo Código General del Proceso ${ }^{12}$, que si bien ordenó al css elaborar un plan de acción que adoptara un nuevo modelo de gestión modificando el modelo procesal de uno escrito a uno oral, hasta la fecha ni se ha pensado en el modelo de gestión para hacer ese giro ni se cuenta con la tecnología, la capacitación, las salas de audiencia necesarias, entre otras. Así, se evidencia el retraso que experimentamos en Colombia frente a estos asuntos de gerencia judicial.

No obstante lo anterior, deben destacarse los esfuerzos de la CEJ para poner este tema en la agenda nacional y para intentar concientizar al csı y a los diseñadores de políticas públicas sobre su relevancia. A pesar de esto, todavía son pocas las personas interesadas en este asunto y aún hay mucho camino por recorrer. Por ello, debe agilizarse el debate en Colombia para estar al día con las nuevas propuestas y para evitar que nuestro sistema judicial se congestione y se deslegitime más de lo que ya está.

\section{E. La importancia de ir más allá de la simple sepa- ración de funciones y su correcta aplicación}

De la revisión de literatura y de las experiencias adelantadas en Colombia se desprende que el enfoque principal, al hablar de modelos de gestión, está en la separación de las funciones administrativas de las jurisdiccionales. Si bien esta es una de las propuestas principales de los

12 Supuestamente entraba a regir el 1 de enero de 2014 sin tener en cuenta si el Plan de Acción ya se había elaborado y se estaba implementando. conocedores del tema, no debe dejarse de lado que la introducción de la gerencia judicial abarca mucho más que la simple separación de funciones; igual de importantes son la implementación de tecnología, el desarrollo de estadística judicial, el manejo del personal, etc.

En este mismo sentido, vale la pena llamar la atención sobre la aplicación incompleta que se ha hecho en Colombia de la propuesta de separar las funciones mediante los centros de servicios judiciales. Cualquier persona que se acerque al centro que funciona en el Complejo Judicial de Paloquemao o al de la sede judicial Hernando Morales, puede comprobar que la única función que están cumpliendo es la radicación de demandas y el reparto respectivo. Así las cosas, la atención de baranda, la consulta de procesos, la radicación de memoriales, la notificación y el manejo de personal aún corresponde a cada despacho judicial. Esta implementación parcial es desafortunada, ya que desvirtúa la propuesta original y puede producir un rechazo de la comunidad hacia la introducción de políticas de gestión que en su parecer realmente no representan mayores beneficios. En consecuencia, se reitera la necesidad de dedicar mayores esfuerzos a estudiar de fondo el tema, a hacer implementaciones completas y planificadas de las propuestas y a generar voluntad política para el progreso de la gerencia judicial.

\section{F. La desactualización del presente debate}

Como última conclusión debe señalarse que, si bien el debate plasmado en este escrito es el que en la actualidad existe y sobre el cual se ha 
escrito, ya está desactualizado y rezagado frente a las últimas propuestas de litigio o juicio en línea. Aquí se hace referencia a un modelo de gestión analógico o de papel, lo cual de ninguna manera implica que el esfuerzo hecho resulte inútil; igual, nuestra justicia es predominantemente escrita y dar el gran paso al litigio en línea es algo que puede demorar. Aun así, no puede perderse de vista que los sistemas judiciales están propendiendo por la justicia en línea y ello implica modificar algunas de las propuestas que hasta el momento se han hecho para reformar los modelos de gestión de los despachos judiciales. Un ejemplo de ello es el hecho de que si el proceso se maneja en línea, la separación de funciones administrativas y jurisdiccionales resulta innecesaria, pues las notificaciones, la atención al usuario, la radicación de demandas y memoriales, la producción de estadística judicial, entre otras, se hará con tan solo un clic. Lo mismo sucedería con la propuesta de aprovechamiento de las economías de escala. Por esta razón, no sólo es ineludible ampliar el conocimiento sobre modelos de gestión y gerencia judicial, sino que también es necesario acelerar el proceso para garantizar un sistema de justicia eficiente, ágil, moderno y de vanguardia.

\section{Referencias}

Arbeláez de Tobón, L. (1996). Modelo de gestión de los juzgados de Itagüí: una alternativa de solución para la justicia colombiana. Recuperado de http://www.oas.org/juridico/spanish/adjust16.htm
Bell, E. (2009). Judicial Case Management. Recuperado de https://docs.google.com/viewe $r ? a=v \& q=$ cache:u1QPnwGM7usJ:www.jsijournal.ie/html/Volume_9_No._2/9[2]_Bell_ Case_management.pdf+\&hl=en\&gl=co\&pid =bl\&srcid=ADGEESjjvpLHj5R1FWUyM-XenOImOVGYDsfYOgNJuvODu2D1J8uOyC--OquDZRrJ1BdZUelyCHY5GmPZszgcEU5YX1Hvs47oCd-VrzqfT_nGIvLIVARzEaJPe2UOTqzhVct LddrSVOq1\&sig=AHIEtbS_sXOoeVXVaBJgWZDfJpyWVKf-bw

Centro de Estudios de Justicia de las Américas. (s. f.). Estudio comparado sobre gestión presupuestaria y gestión administrativa de cortes y tribunales y tratamiento estadístico de la información sobre el funcionamiento del sistema judicial. Recuperado de http://www.cejamericas.org/webapp/index. php?searchword=gesti\%C3\%B3n\%20presup uestar\&ordering=newest \&searchphrase=all \&limit=20\&option=com_search

Consejo Superior de la Judicatura. (Diciembre de 2012). Plan de Acción para la Implementación del Código General del Proceso. Recuperado de http://www.google.com.co/url ? sa $=\mathrm{t} \& \mathrm{rct}=\mathrm{j} \& \mathrm{q}=\& \mathrm{esrc}=\mathrm{s} \&$ source $=$ web $\& \mathrm{~cd}=$ $3 \&$ ved $=0$ CEAQFjAC\&url=http $\% 3 A \% 2 F \% 2 F w$ ww.ramajudicial.gov.co\%2Fcsj\%2Fdownload $\mathrm{s} \% 2$ FarchivosEventos\%2F5840.doc\&ei=PuR tUqnMJNLfkQeT3YCwAQ\&usg=AFQjCNGGE6 li_cwrX-Jba21Si4M6wEVA1g\&sig2=-2uajlvOT 39KdF2qYVPxTg\&bvm=bv.55123115,d.eWO

Consejo Superior de la Judicatura. (noviembre de 2010). Plan sectorial de desarrollo de la 
rama judicial 2011-2014. Hacia una justicia eficiente, un propósito nacional. Recuperado de http://200.74.129.92/ModeloCSJ/ documentos_portal/PIan\%20Sectorial\%20 2011\%20-\%202014.pdf

Consejo Superior de la Judicatura. (s. f.). Modernización de los modelos de gestión en los despachos judiciales de la especialidad civil. Recuperado de http://www.google.com.co/ url?sa $=\mathrm{t} \& \mathrm{rct}=\mathrm{j} \& \mathrm{q}=\& \mathrm{esrc}=\mathrm{s} \& \mathrm{source}=\mathrm{we}$ \& $\& \mathrm{~cd}$ $=1 \& \mathrm{ved}=0 \mathrm{CCOQFjAA} \& \mathrm{url}=\mathrm{http} \% 3 \mathrm{~A} \% 2 \mathrm{~F} \% 2 \mathrm{Fw}$ ww.ramajudicial.gov.co\%2Fcsj\%2Fdownloads \%2FUserFiles\%2FFile\%2FCSJ\%2FDESARROL LOS\%2520CON\%2520CREDITO\%2520Y\%25 20COOPERACION\%2520INTERNACIONAL[1]. pdf\&ei=zXI1UZeICoKE9QSbxIGIDw\&usg=AFQ jCNHVZoNI8ejxQr_ezO6LJXqYuo5zHQ\&sig2=I ELmTRMzlkIMULmp7CkrfQ\&bvm=bv.431489 75,d.eWU

Contreras, L. M. (s. f.). Oficina judicial: la organización de los tribunales y la nueva oficina judicial. Recuperado de http://www.cej-mjusticia.es/cej_dode/doc_users/pdf/nueva_oficina_judicial/NOJ/OFICINA_JUDICIAL_Martin_Contreras.pdf

Cornejo Valdivia, R. (Octubre 5 y 6 de 2005). Una visión global de la gestión del despacho judicial en Perú. III Seminario Interamericano de Gestión Judicial. Managua, Nicaragua. Recuperado de http://www.cejamericas.org/ doc/eventos/per-cornejo-vision2.pps

El Tiempo. (8 de julio de 2013). El 56\% cree que la corrupción empeoró en los últimos dos años. Recuperado de www.eltiempo.com/ justicia/ARTICULO-WEB-NEW_NOTA_INTERIOR-12920062.html

Garavano, G. C., Chayer, H. M., Cambellotti, C. A. y Ricci, M. (3 de septiembre de 1999). La problemática de la oficina judicial en la Argentina. El Derecho, xxxVII (9828).

García Villegas, M. y Sousa Santos, B. (2001). El caleidoscopio de las justicias en Colombia: análisis socio-jurídico. Bogotá: Siglo del Hombre Editores y Universidad de los Andes.

Giraldo Estrada, D. (2013). Entrevista personal.

Guerra Cerrón, M. E. (2002). A propósito de la reforma judicial peruana. Seminario internacional: Experiencias de Reformas a la Gestión Judicial. Recuperado de: http://www.cejamericas.org/doc/documentos/prop_ref_jud.pdf

Guerra Cerrón, M. E. (2002). El modelo corporativo: Origen y objetivo. Recuperado de https://docs.google.com/ viewer? $a=v \& q=$ cache:33DMRXI-r9EJ:www. cejamericas.org/index.php/biblioteca/biblioteca-virtual/doc_view/689-el-modelo-corporativo-origen-y-objetivo+\%22El+modelo+cor porativo:+Origen+y+objetivo $\% 22 \& \mathrm{hl}=e n \& p$ $i d=b \mid \& s r c i d=A D G E E S j 50 V e H m f W u y D 4 U i l f X$ LSIHNKRva_wD8I71jol_OXTE5yav03ZQGnT wVCXctzfO2EUyc68SFDd7Ju6_BOGZjn9vwrnPOmhEIOcyTiF-Un_S-ERZrZONnapM_LYOmZ PkhEz34AK3\&sig=AHIEtbQWRmYbGRpYVgp Wvtl2ihr19aJbQg 
Hall, D. J., Hoffman, R. B. y Stromsen, J. M. (2003). Professional Court Administration: The key to Judicial Independence. Recuperado de http://cdm16501.contentdm.oclc. org/cdm/ref/collection/judicial/id/204

Hitt, M., Black, S. y Porter, L. (2006). Administración. México: Pearson Education.

Hoyle, D. (2009). ISO 9000 Quality Sistems Handbook. Gran Bretaña: Elsevier's Science and Technology.

International Consortium for Court Excellence, (2013). International Framework for Court Excellence. Recuperado de http://www.courtexcellence.com/Resources/The-Framework. aspx

Lienhard, A. y Kettinger, D. (Enero de 2011). Research on the caseload management of courts: methodological questions. Recuperado de https://docs.google.com/viewer?a=v $\& q=$ cache:xHeovdNh7TEJ:www.utrechtlawreview.org/index.php/ulr/article/view/147/1 $46+\&$ hl=en $\& g|=c o \& p i d=b| \& s r c i d=A D G E E S$ h7di9PHLiL7GBy-_ottKFTaOaMPNtCF7IxWiBkfkcHw-xBkGzqbIS_o_AfXfoBoqDg3GEPCmhhqUbRcA4E1m0E-fDx8AI1M6xuiYBqfv_ hLT60TLuTPm6aXkcELOVXD4Hsf5gH\&sig=A HIEtbSzdZmowgE4HelOOYAx_SxrxpCkhw

Londoño Sepúlveda, N. R. (Enero-Junio de 2010). El uso de las TIC en el proceso judicial: una propuesta de justicia en línea. Revista Facultad de Derecho y Ciencias Políticas, 40, 112.
López Medina, D. E. (22 de mayo de 2000). Nuevas tendencias en la dirección judicial del proceso. Recuperado de http://www.ramajudicial.gov.co/csj/downloads/UserFiles/File/ CALDAS/INFORMES\%20CSJ/GESTION\%20 DE\%2OLA\%20FORMACION\%20JUDICIAL/ Nuevas_Tendencias_en_la_Direccion_Judicial_del_proceso\%20-\%20copia.pdf

Mann, B. (Agosto de 2009). Past and future for management of courts. Recuperado de LWB https://docs.google.com/viewer? $a=v \& q=$ cache: $d$ mcBbULpeTAJ:www.iaca. ws/files/LWB-PastAndFutureManagmentOfCourts. $p d f+\& h l=e n \& g l=c o \& p i d=b \mid \& s r c i d=-$ ADGEESg18zTAGT07QBCj8uKszIzI4YM62 XkRJCs2OaBNoc6XhgPwvxJU3RIsqW8bSmWa-YYPpxlhighOJY6XRnS4rKxW6d8clyn9 QPR c Zmy HxvOk 9 OLUxorhkWXA_ eyDIundIPfyIVNQ\&sig=AHIEtbSIEa-UtVLiKCz2RzJ6nPMFf9sVw

Ministerio de Justicia de Nueva Zelanda. Knowledge management defined [en línea]. Consultado el 7 de noviembre de 2013. Recuperado de http://www.justice.govt.nz/ justice-sector/strategy/justice-sector-information-strategy/publications/informationknowledge-management-guide/chapter-2knowledge-management-defined

Morales, H. (1965). Curso de derecho procesal civil. Bogotá: El Gráfico Editores Ltda.

National Association for Court Management. (1992). The court Administrator: A Manual. Recuperado de https://docs.google.com/ 
viewer?a $=v \& q=$ cache:dmnuztObyfOJ:www. fjc.gov/public/pdf.nsf/lookup/court_admin. pdf/\%24file/court_admin.pdf $+\& h l=e n \& g l=c$ o\&pid=bl\&srcid=ADGEESjxgeScPaFCDpdl4IOrPScaiJ6mlo35Z0mv4wLw9oXa7dhTZiit4gUiCnUei6QP9Fr30UbGdf9yRoi85RiQRnBdZp8dJsC2yh0jRAB_DAsJhta31dHmp3PSEkxW8b_tA7mm04N\&sig=AHIEtbRYgjFJ9Y7UL 8meiSHwk4W6eorSKg

Navarro Solano, S. (s. f.). Programa de mejoramiento de la Administración de justicia en Costa Rica. Sistema Judiciales: ¿Crisis en la capacitación judicial?, 1, p. 125-131. Recuperado de http://issuu.com/sistemasjudiciales/docs/sistemasjudiciales1/125

Ochoa Cruz, P. (s. f.). Juicio en Line@: La experiencia del Tribunal Federal de Justicia Fiscal y Administrativa en México. Recuperado de http://www.cejamericas.org/ponencias_XSemgestionjudicial/Panel10/Pochoa_TFJFA_ Mexico.pdf

Organización Internacional de Normalización (ISO). (2005). Norma internacional ISO 9000: Sistemas de gestión de calidad - Fundamentos y vocabulario. Recuperado de http://www. google.com.co/url?sa=t\&rct=j\&q=\&esrc=s\&s ource $=$ web $\& c d=1 \&$ sqi $=2 \&$ ved $=0$ CB $8 Q F j A A \&$ url=http\%3A\%2F\%2Fwww.rree.go.cr\%2Ffiledd.php\%3Fid_file\%3D340\&ei=mWZzUIzFMI b68QTChoCQBA\&usg=AFQjCNGFxiFkKtQc2s pw3k2eFlp8jnS2A\&sig2=21R2XtElugo_IUmBXyOxxw.

Pandey, A. (30 de mayo de 2011). An Emerging Trend in Judiciary: Court Management.
Recuperado de MigthyLaws: http://www. mightylaws.in/660/emerging-trend-judiciarycourt-management

Pérez-Pujazón Millán, E. y Trigo y Sierra, E. (2010). La reciente reforma de la Ley de Enjuiciamiento civil para la implantación de la nueva oficina judicial. Recuperado de http://www. uria.com/documentos/publicaciones/2513/ documento/articuloUM.pdf?id=3034

Quiroga Lavié, H. (1998). La formación del derecho procesal organizacional. Argentina, Buenos Aires: Cámara de Diputados de la Provincia de Buenos Aires.

Rivas Acuña, I. (2007). Los conceptos, teorías y modelos de la administración judicial en el estado constitucional. Recuperado de http:// www.juridicas.unam.mx/publica/librev/rev/ refjud/cont/9/rjf/rjf5.pdf

Santos, P. (Julio de 2003). Los nuevos sistemas de organización y gestión de la justicia: ¿mito o realidad? Recuperado de http://webcache. googleusercontent.com/search?q=cache:ywmY300rQQJ:accesoalajusticia.org/documentos/getbindata.php?dcfid\%3D73+Pasto $\mathrm{r}+$ Santos\%2B+los+nuevos+sistemas+de+or ganizaci\%C3\%B3n+y+gesti\%C3\%B3n+de+la +justicia:+\%C2\%BFmito+o+realidad?\%22\&h I=en

Singapore Judiciary's Integrated Electronic Litigation System. (s. f.). E-litigation. Recuperado de https://www.elitigation.sg/index.aspx 
Slowes, R. (2012). Benefits of a Modern Court Case Management System. Recuperado de http://thomsonreuters.com/products/ legal/c-track-court-case-mgmt/benefits-of-amodern-CMS-rick-slowes.pdf

Solomon, H. (Ed.). (2002). Developing a Court Leadership and Management Curriculum. Recuperado de http://jeritt.msu.edu/monographs/JERITT\%20Monograph\%2013.pdf

Terán y Contreras, J. M. (2012). Juicio en línea en la justicia administrativa: hacia la justicia electrónica en México. Recuperado de http:// www.azc.uam.mx/publicaciones/alegatos/ pdfs/73/80-02.pdf

International Consortium for Court Excellence. (2013). The International Framework for Court Excellence. Recuperado de http://www.courtexcellence.com/Resources/ /media/Microsites/Files/ICCE/The\%20International\%20 Framework\%2021E\%202013.ashx

Vargas Vianco, J. E. (Septiembre de 2005). Herramientas para el diseño de despachos judiciales. Recuperado de http:// www.cejamericas.org/webapp/index. php?searchword=dise $\%$ C3\%B10\%20de\%20 despachos\&ordering=newest \&searchphrase =all\&limit=20\&option=com_search

Vega Robert, R. (18 de julio de 2008). Carta al doctor Luis Paulino Mora Mora Recuperado de: https://docs.google.com/viewer?attid=0. $1 \&$ pid=gmail\&thid=13a41dddeba41ad6\&url =https://mail.google.com/mail/u/0/?ui\%3D
2\%26ik\%3D37da5e992a\%26view\%3Datt\%2 6th\%3D13a41dddeba41ad6\%26attid\%3D0 .1\%26disp\%3Dsafe\%26realattid\%3Df_h81z 73wh5\%26zw\&docid=f9e993e135603ed6c dfcad731447bab7|8ac35c4303ecfbda519 f130612adc43a\&chan=EAAAABtuGksK8sN wPFWxumzXRRUiRBAXReo/1/aCIWvsTHtX\&a =v\&rel=zip;z21;Costa+Rica+CORTE+PLENA. MEGASDESPACHOS3.doc

Weske, M. (2012). Business, Process, Management: Concepts, Languages, Architectures. New York: Springer.

\section{ANEXO}

\section{Bibliografía sugerida}

\section{Para conocer la situación de la justicia en Colom-} bia:

Consejo Superior de la Judicatura. (s. f.). Informes anuales al Congreso de la República. de: http://www.ramajudicial.gov.co/csj/publicaciones/index/subcategoria/359/924/ Informe-al-Congreso-de-la-Rep\%C3\%BAblica

Corporación Excelencia de la Justicia. (s. f.). Indicadores generales de justicia en Colombia. Recuperado de http://www.cej.org.co/index. php/indicadores-de-justicia

García Villegas, M. y Sousa Santos, B. (2001). El caleidoscopio de las justicias en Colombia: Análisis socio-jurídico. Bogotá: Siglo del Hombre Editores y Universidad de los Andes. 
El Tiempo. (8 de julio de 2013). El 56\% cree que la corrupción empeoró en los últimos dos años. Recuperado de www.eltiempo.com/ justicia/ARTICULO-WEB-NEW_NOTA_INTERIOR-12920062.html

Para entender en qué consiste un sistema de gestión de calidad desde una perspectiva de la administración de empresas:

Hitt, M., Black, S. y Porter, L. (2006). Administración. México: Pearson Education.

Hoyle, D. (2009). ISO 9000 Quality Sistems Handbook. Gran Bretaña: Elsevier`s Science and Technology.

Organización Internacional de Normalización (ISO). (2005). Norma internacional ISO 9000: Sistemas de gestión de calidad - Fundamentos y vocabulario. Recuperado de de http:// www.google.com.co/url?sa $=t \& r c t=j \& q=\& e$ $\mathrm{src}=\mathrm{s} \&$ so $\mathrm{rce}=$ we $b \mathrm{~cd}=1 \& \mathrm{sq} i=2 \& \mathrm{ved}=0$ CB8QFjAA\&url=http\%3A\%2F\%2Fwww.rree. go.cr\%2Ffile-dd.php\%3Fid_file\%3D340\&ei= mWZzUIzFMIb68QTChoCQBA\&usg=AFQjCNG FxiFkKtQc2spw3k2eFIp8jnS2A\&sig2=21R2X tElugo_IUmBXyOxxw.

Weske, M. (2012). Business, Process, Management: Concepts, Languages, Architectures. New York: Springer.
Para comprender la discusión sobre gerencia judicial y el diseño de nuevos modelos de gestión de los despachos judiciales:

Garavano, G. C., Chayer, H. M., Cambellotti, C. A. y Ricci, M. (3 de septiembre de 1999). La problemática de la oficina judicial en la Argentina. El Derecho, xxxvII (9828).

Centro de Estudios de Justicia de las Américas. (s.f.). Estudio comparado sobre gestión presupuestaria y gestión administrativa de cortes y tribunales y tratamiento estadístico de la información sobre el funcionamiento del sistema judicial. Recuperado de http://www.cejamericas.org/webapp/index. php?searchword=gesti\%C3\%B3n\%20presup uestar\&ordering=newest\&searchphrase=all \&limit=20\&option=com_search

Hall, D. J., Hoffman, R. B. y Stromsen, J. M. (2003). Professional Court Administration: The key to Judicial Independence. Recuperado de http://cdm16501.contentdm.oclc. org/cdm/ref/collection/judicial/id/204

International Consortium for Court Excellence. (2013). International Framework for Court Excellence. Recuperado de http://www.courtexcellence.com/Resources/The-Framework. aspx

Mann, B. (Agosto de 2009). Past and future for management of courts. Recuperado de LWB: https://docs.google.com/viewer $? a=v \& q=$ cache: $d$ mcBbULpeTAJ:www.iaca. ws/files/LWB-PastAndFutureManagmentO- 
fCourts.pdf $+\& h|=e n \& g|=c o \& p i d=b \mid \& s r c i d=-$ ADGEESg18zTAGT07QBCj8uKszIzI4YM62 XkRJCs20aBNoc6XhgPwvxJU3RIsqW8bSmWa-YYPpxlhighOJY6XRnS4rKxW6d8clyn9 Q PR c ZmyHxvOk9OLUxorhkWXA_ eyDIundIPfyIVNQ\&sig=AHIEtbSIEa-UtVLiKCz2RzJ6nPMFf9sVw

Ministerio de Justicia de Nueva Zelanda. Knowledge management defined. Recuperado de http://www.justice.govt.nz/justicesector/strategy/justice-sector-informationstrategy/publications/information-knowledge-management-guide/chapter-2-knowledgemanagement-defined

Pandey, A. (30 de mayo de 2011). An Emerging Trend in Judiciary: Court Management. Recuperado de MigthyLaws: http://www. mightylaws.in/660/emerging-trend-judiciarycourt-management

Rivas Acuña, I. (2007). Los conceptos, teorías y modelos de la administración judicial en el estado constitucional. Recuperado de http:// www.juridicas.unam.mx/publica/librev/rev/ refjud/cont/9/rif/rjf5.pdf

Santos, P. (Julio de 2003). Los nuevos sistemas de organización y gestión de la justicia: ¿mito o realidad? Recuperado de http://webcache. googleusercontent.com/search?q=cache:ywmY300rQQJ:accesoalajusticia.org/documentos/getbindata.php?dcfid\%3D73+Pasto $r+$ Santos\%2B+los+nuevos+sistemas+de+or ganizaci\%C3\%B3n+y+gesti $\%$ C3\%B3n+de+la +justicia:+\%C2\%BFmito+o+realidad?\%22\&h I=en
Vargas Vianco, J. E. (Septiembre de 2005). Herramientas para el diseño de despachos judiciales. Recuperado de http://sistemasjudiciales.org/content/jud/archivos/notaarchivo/422.pdf

Para conocer textos en donde se ha mencionado el derecho procesal organizacional:

Garavano, G. C., Chayer, H. M., Cambellotti, C. A. y Ricci, M. (3 de septiembre de 1999). La problemática de la oficina judicial en la Argentina. El Derecho, xxxvII (9828).

Quiroga Lavié, H. (1998). La formación del derecho procesal organizacional. Argentina, Buenos Aires: Cámara de Diputados de la Provincia de Buenos Aires.

Para información sobre uno de los componentes de la gestión de la administración de justicia, cuál es la gestión de la carga de trabajo o asignación de casos dependiendo de la dificultad del caso, el tiempo requerido para su solución y la disponibilidad de tiempo de los jueces:

Lienhard, A. y Kettinger, D. (Enero de 2011). Research on the caseload management of courts: methodological questions. Recuperado de https://docs.google.com/viewer?a=v $\& q=$ cache:xHeovdNh7TEJ:www.utrechtlawreview.org/index.php/ulr/article/view/147/1 $46+\& h l=e n \& g l=c o \& p i d=b \mid \& s r c i d=A D G E E S$ h7di9PHLiL7GBy-_ottKFTaOaMPNtCF7IxWiBkfkcHw-xBkGzqbIS_o_AfXfoBoqDg3GEPCmhhqUbRcA4E1m0E-fDx8Al1M6xuiYBqfv_ hLT60TLuTPm6aXkcELOVXD4Hsf5gH\&sig=A HIEtbSzdZmowgE4HelOOYAx_SxrxpCkhw 
Slowes, R. (2012). Benefits of a Modern Court Case Management System. Recuperado de: http://thomsonreuters.com/products/ legal/c-track-court-case-mgmt/benefits-of-amodern-CMS-rick-slowes.pdf

Vargas Vianco, J. E. (Septiembre de 2005). Herramientas para el diseño de despachos judiciales. Recuperado de http://sistemasjudiciales.org/content/jud/archivos/notaarchivo/422.pdf

Sobre los administradores de los despachos judiciales:

National Association for Court Management. (1992). The court administrator: A Manual. Recuperado de https://docs.google.com/ viewer?a=v\&q=cache:dmnuztObyfOJ:www. fjc.gov/public/pdf.nsf/lookup/court_admin. pdf/\%24file/court_admin.pdf+\&hl=en\&gl=c o\&pid=bl\&srcid=ADGEESjxgeScPaFCDpdl4IOrPScaiJ6mlo35Z0mv4wLw9oXa7dhTZiit4gUiCnUei6QP9Fr30UbGdf9yRoi85RiQRnBdZp8dJsC2yh0jRAB_DAsJhta31dHmp3PSEkxW8b_tA7mm04N\&sig=AHIEtbRYgjFJ9Y7UL 8meiSHwk4W6eorSKg

Vargas Vianco, J. E. (Septiembre de 2005). Herramientas para el diseño de despachos judiciales. Recuperado de http://sistemasjudiciales.org/content/jud/archivos/notaarchivo/422.pdf

Santos, P. (Julio de 2003). Los nuevos sistemas de organización y gestión de la justicia: ¿mito o realidad? Recuperado de http://webcache. googleusercontent.com/search?q=cache:ywmY300rQQJ:accesoalajusticia.org/documentos/getbindata.php?dcfid\%3D73+Pasto $\mathrm{r}+$ Santos $\% 2 \mathrm{~B}+$ los + nuevos+sistemas+de+or ganizaci\%C3\%B3n+y+gesti\%C3\%B3n+de+la +justicia:+\%C2\%BFmito+o+realidad?\%22\&h I=en

\section{Sobre la discusión anglosajona de los modelos de gestión:}

Bell, E. (2009). Judicial Case Management. Recuperado de https://docs.google.com/viewe $r ? a=v \& q=$ cache:u1QPnwGM7usJ:www.jsijournal.ie/html/Volume_9_No._2/9[2]_Bell_ Case_management.pdf $+\& \mathrm{hl}=\mathrm{en} \& g \mathrm{l}=\mathrm{co} \& \mathrm{pid}$ $=$ bl\&srcid=ADGEESjjvpLHj5R1FWUyM-XenOImOVGYDsfYOgNJuvODu2D1J8uOyC--OquDZRrJ1BdZUelyCHY5GmPZszgcEU5YX1Hvs47oCd-VrzqfT_nGIvLIVARzEaJPe2UOTqzhVct LddrSVOq1\&sig=AHIEtbS_sX0oeVXVaBJgWZDfJpyWVKf-bw

Solomon, H. (Ed.). (2002). Developing a Court Leadership and Management Curriculum. Recuperado de http://jeritt.msu.edu/monographs/JERITT\%20Monograph\%2013.pdf

\section{Sobre la experiencia de Costa Rica con la intro- ducción de la gestión judicial:}

Navarro Solano, S. (s. f.). Programa de mejoramiento de la administración de justicia en Costa Rica. Sistemas judiciales: ¿crisis en la capacitación judicial?, 1, p. 125-131. Recuperado de http://issuu.com/sistemasjudiciales/docs/sistemasjudiciales1/125 
Vega Robert, R. (18 de julio de 2008). Carta al doctor Luis Paulino Mora Mora. Recuperado de https://docs.google.com/viewer?attid=0. 1\&pid=gmail\&thid=13a41dddeba41ad6\&url =https://mail.google.com/mail/u/0/?ui\%3D 2\%26ik\%3D37da5e992a\%26view\%3Datt\%2 6th\%3D13a41dddeba41ad6\%26attid\%3D0 .1\%26disp\%3Dsafe\%26realattid\%3Df_h81z 73wh5\%26zw\&docid=f9e993e135603ed6c dfcad731447bab7|8ac35c4303ecfbda519 f130612adc43a\&chan=EAAAABtuGksK8sN wPFWxumzXRRUiRBAXReo/1/aCIWvsTHtX\&a =v\&rel=zip;z21;Costa+Rica+CORTE+PLENA. MEGASDESPACHOS3.doc

\section{Sobre la experiencia de Perú con la introducción de la gestión judicial:}

Cornejo Valdivia, R. (Octubre 5 y 6 de 2005). Una visión global de la gestión del despacho judicial en Perú. III Seminario Interamericano de Gestión Judicial. Managua, Nicaragua. Recuperado de http://www.cejamericas.org/ doc/eventos/per-cornejo-vision2.pps

Guerra Cerrón, M. E. (2002). A propósito de la reforma judicial peruana. Seminario internacional: Experiencias de Reformas a la Gestión Judicial. Recuperado de http://www.cejamericas.org/doc/documentos/prop_ref_jud.pdf

Guerra Cerrón, M. E. (2002). El modelo corporativo: origen y objetivo. Recuperado de https://docs.google.com/ viewer?a=v\&q=cache:33DMRXI-r9EJ:www. cejamericas.org/index.php/biblioteca/biblioteca-virtual/doc_view/689-el-modelo-corpo-
rativo-origen-y-objetivo+\%22EI+modelo+cor porativo:+Origen+y+objetivo $\% 22 \& \mathrm{hl}=e n \& p$ $i d=b l \& s r c i d=A D G E E S j 50 V e H m f W u y D 4 U i l f X$ LSIHNKRva_wD8I71jol_OXTE5yav03ZQGnT wVCXctzfO2EUyc68SFDd7Ju6_BOGZjn9vwrnPOmhEIOcyTiF-Un_S-ERZrZONnapM_LYOmZ PkhEz34AK3\&sig=AHIEtbQWRmYbGRpYVgp Wvtl2ihr19aJbQg

\section{Sobre la experiencia de España con la introducción} de la gestión judicial:

Contreras, L. M. (s. f.). Oficina judicial: la organización de los tribunales y la nueva oficina judicial. Recuperado de: http://www.cejmjusticia.es/cej_dode/doc_users/pdf/nueva_oficina_judicial/NOJ/OFICINA_JUDICIAL_ Martin_Contreras.pdf

Pérez-Pujazón Millán, E. y Trigo y Sierra, E. (2010). La reciente reforma de la Ley de Enjuiciamiento civil para la implantación de la nueva oficina judicial. Recuperado de: http://www. uria.com/documentos/publicaciones/2513/ documento/articuloUM.pdf?id=3034

Para descubrir lo que se ha escrito sobre modelos de gestión de los despachos judiciales en Colombia y las experiencias de algunos juzgados:

Arbeláez de Tobón, L. (1996). Modelo de gestión de los juzgados de Itagüí: una alternativa de solución para la justicia colombiana. Recuperado de http://www.oas.org/juridico/spanish/adjust16.htm

Consejo Superior de la Judicatura. (Diciembre de 2012). Plan de Acción para la Implemen- 
tación del Código General del Proceso. Recuperado de http://www.google.com.co/url ?sa $=\mathrm{t} \& \mathrm{rct}=\mathrm{j} \& \mathrm{q}=\& \mathrm{esrc}=\mathrm{s} \& \mathrm{source}=\mathrm{web} \& \mathrm{~cd}=$ $3 \&$ ved $=0 C E A Q F j A C \& u r l=h t t p \% 3 A \% 2 F \% 2 F w$ ww.ramajudicial.gov.co\%2Fcsj\%2Fdownload s\%2FarchivosEventos\%2F5840.doc\&ei=PuR tUqnMJNLfkQeT3YCwAQ\&usg=AFQjCNGGE6 li_cwrX-Jba21Si4M6wEVA1g\&sig2=-2uajlvOT 39KdF2qYVPxTg\&bvm=bv.55123115,d.eWO

Corporación Excelencia en la Justicia. (Junio 30 de 2011). Propuesta de ajuste al modelo de gestión de los juzgados civiles municipales de Bogotá D.C. Documento explicativo.n

López Medina, D. E. (22 de mayo 22 de 2000). Nuevas tendencias en la dirección judicial del proceso. Recuperado de: http://www.ramajudicial.gov.co/csj/downloads/UserFiles/File/ CALDAS/INFORMES\%20CSJ/GESTION\%20 DE\%20LA\%2OFORMACION\%20JUDICIAL/ Nuevas_Tendencias_en_la_Direccion_Judicial_del_proceso\%20-\%20copia.pdf

Para conocer el discurso del Consejo Superior de la Judicatura respecto a los modelos de gestión de los despachos judiciales:

Consejo Superior de la Judicatura. (s. f.). Modernización de los modelos de gestión en los despachos judiciales de la especialidad civil. Recuperado de http://www.google.com.co/ url?sa $=\mathrm{t} \& \mathrm{rct}=\mathrm{j} \& \mathrm{q}=\& \mathrm{esrc}=\mathrm{s} \& \mathrm{source}=\mathrm{web} \& \mathrm{~cd}$ $=1 \&$ ved $=0 C C 0 Q F j A A \&$ url=http $\% 3 A \% 2 F \% 2 F w$ ww.ramajudicial.gov.co\%2Fcsj\%2Fdownloads \%2FUserFiles\%2FFile\%2FCSJ\%2FDESARROL LOS\%2520CON\%2520CREDITO\%2520Y\%25
20COOPERACION\%2520INTERNACIONAL[1]. pdf\&ei=zXI1UZelCoKE9QSbxIGIDw\&usg=AFQ jCNHVZoNI8ejxQr_ezO6LJXqYuo5zHQ\&sig2=I ELmTRMzIkIMULmp7CkrfQ\&bvm=bv.431489 75,d.eWU

Consejo Superior de la Judicatura. (Diciembre de 2012). Plan de Acción para la Implementación del Código General del Proceso. Recuperado de http://www.google.com.co/url ?sa $=\mathrm{t} \& \mathrm{rct}=\mathrm{j} \& \mathrm{q}=\& \mathrm{esrc}=\mathrm{s} \&$ source $=\mathrm{web} \& \mathrm{~cd}=$ $3 \&$ ved $=0$ CEAQFjAC\&url=http $\% 3 A \% 2 F \% 2 F w$ ww.ramajudicial.gov.co\%2Fcsj\%2Fdownload s\%2FarchivosEventos\%2F5840.doc\&ei=PuR tUqnMJNLfkQeT3YCwAQ\&usg=AFQjCNGGE6 li_cwrX-Jba21Si4M6wEVA1g\&sig2=-2uajlvOT 39KdF2qYVPxTg\&bvm=bv.55123115,d.eWO

Consejo Superior de la Judicatura. (Noviembre de 2010). Plan sectorial de desarrollo de la rama judicial 2011-2014. Hacia una justicia eficiente, un propósito nacional. Recuperado de http://200.74.129.92/ModeloCSJ/ documentos_portal/Plan\%20Sectorial\%20 2011\%20-\%202014.pdf

\section{Respecto al uso de las TIC en el proceso judicial:}

Londoño Sepúlveda, N. R. (Enero-Junio de 2010). El uso de las TIC en el proceso judicial: una propuesta de justicia en línea. Revista Facultad de Derecho y Ciencias Políticas, 40, 112.

\section{Sobre el derecho procesal civil:}

Morales, H. (1965). Curso de derecho procesal civil. Bogotá: El Gráfico Editores Ltda. 
En cuanto al litigio, juicio o proceso en línea, puede consultarse:

Londoño Sepúlveda, N. R. (Enero-Junio de 2010). El uso de las TIC en el proceso judicial: una propuesta de justicia en línea. Revista Facultad de Derecho y Ciencias Políticas, 40, 112.

Ochoa Cruz, P. (s. f.). Juicio en Line@: la experiencia del Tribunal Federal de Justicia Fiscal y Administrativa en México. Recuperado de http://www.cejamericas.org/ponencias_XSe-
mgestionjudicial/Panel10/Pochoa_TFJFA_ Mexico.pdf

Singapore Judiciary's Integrated Electronic Litigation System. (s. f.). E-litigation. Recuperado de https://www.elitigation.sg/index.aspx

Terán y Contreras, J. M. (2012). Juicio en línea en la justicia administrativa: hacia la justicia electrónica en México. Recuperado de http:// www.azc.uam.mx/publicaciones/alegatos/ pdfs/73/80-02.pdf 